\title{
Saving 2 lives with 1 operation!
}

\author{
Nicholas G. Smedira, MD
}

From the Department of Thoracic/Cardiovascular Surgery, Cleveland Clinic Foundation, Cleveland, Ohio. Disclosures: Author has nothing to disclose with regard to commercial support.

Received for publication Jan 7, 2016; accepted for publication Jan 8, 2016; available ahead of print Feb 17, 2016.

Address for reprints: Nicholas G. Smedira, MD, Department of Thoracic/Cardiovascular Surgery, Cleveland

Clinic Foundation, Cleveland, OH 44195 (E-mail: smedirn@ @cf.org).

J Thorac Cardiovasc Surg 2016;151:1161-2

$0022-5223 / \$ 36.00$

Copyright (c) 2016 by The American Association for Thoracic Surgery

http://dx.doi.org/10.1016/j.jtcvs.2016.01.015

Managing extracorporeal life support has numerous challenges, but complicating matters with the need to save both the mother and the fetus raise the bar to new heights. Although it is uncommon, centers specializing in the use of extracorporeal membrane oxygenation (ECMO) will at one time face the need to support a patient while she is pregnant. Moore and colleagues ${ }^{1}$ have reviewed the literature, finding 45 cases of ECMO support during pregnancy. The survival for both the mother and the fetus was surprisingly high at $78 \%$ and $65 \%$, respectively. The majority of patients were supported with veno-veno systems, and bleeding was the main cause of death.

The major contribution of this meta-analysis is in providing strong support for an extremely aggressive approach for pregnant woman with severe cardiopulmonary dysfunction. What is lacking, but was not the authors' focus, is how to maximize survival. The remainder of this editorial will discuss the clinical and technical considerations in patient management.

The physiologic changes that occur during pregnancy have pushed cardiopulmonary function to near maximal levels such that any additional insult is poorly tolerated. Cardiac output increases by $50 \%$ to meet the metabolic needs of the mother and fetus. This is accomplished by a rightward shift along the Starling curve, an increase in heart rate, and a lowering of the systemic vascular resistance. Blood volume increases by $45 \%$ with a decrease in hematocrit, and the enlarging uterus reduces residual lung capacity by at least $20 \%$. Blood $\mathrm{pH}$ is maintained near normal but with a respiratory alkalosis compensated by a bicarbonate losing metabolic acidosis. With all these changes, any further decline in cardiac or pulmonary function results in severe physiologic compromise. Finally, pregnancy is thought to induce a hypercoagulable state with an increase in fibrinogen and most other coagulation factors and a decrease in protein $S$.

The decision matrix for extracorporeal support requires rapid assessment of cardiopulmonary function involving multidisciplinary collaboration, including critical care teams, maternal-fetal medicine physicians, perfusion services, and cardiothoracic surgery. The initial

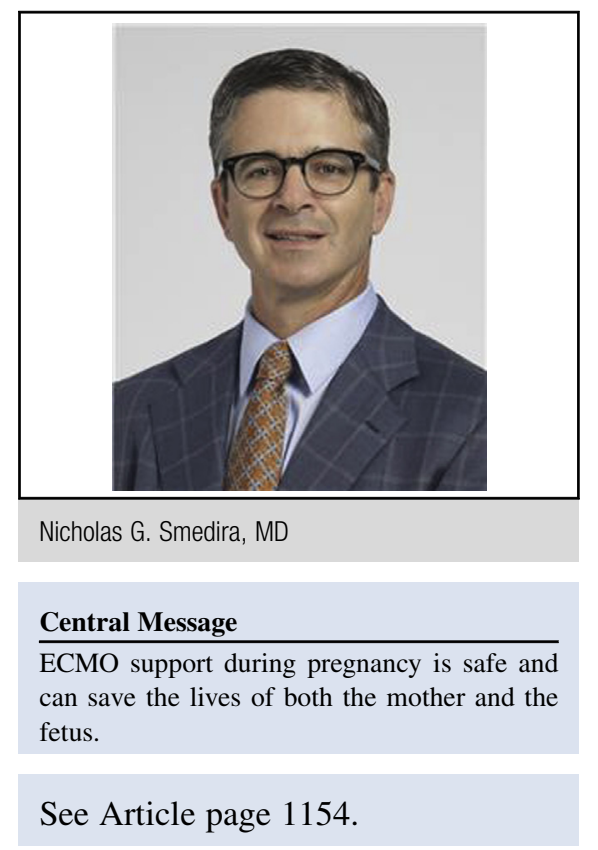

step in the decision process centers on whether there is adequate cardiac function to meet the metabolic needs of the patient and fetus. Pulmonary artery catheter determination of cardiac output by both thermodilution and Fick calculation, along with transthoracic echocardiography, guides the decision for venoarterial or veno-veno support.

ECMO cannula and circuit capabilities, best assessed with an experienced perfusionist, are now compared with the physiologic data to determine where to access the circulation and how much flow will be needed. Severe cardiopulmonary dysfunction requires venoarterial flow to match metabolic needs. Although seemingly simple, vascular access that provides high flow while avoiding limb ischemia or hyperperfusion and is not a source of ongoing bleeding can be extremely challenging to accomplish. For patients with mild cardiac dysfunction (usually right ventricular) secondary to hypoxia, acidosis, and hypercarbia, the use of lower-extremity venoarterial ECMO can result in profound cerebral and cardiac hypoxia because the ECMO system's maximum flow capacity of $4 \mathrm{~L} / \mathrm{min}$ will not completely eliminate pulmonary blood flow and left ventricular ejection of desaturated blood.

For isolated severe lung injury, which was the most common indication for ECMO in this series, veno-veno ECMO support is the safest and most effective. Many centers are now choosing a right internal jugular approach 
using a single double-lumen cannula (Avalon Elite; Maquet, Rastatt, Germany). ${ }^{1}$ This cannula drains venous blood from both cava and returns it to the right atrium. Both transesophageal echocardiography and fluoroscopy have been used to guide placement. Mobilization of the patient, including ambulation, is possible, and there is much less bleeding compared with venoarterial cannulation. Anticoagulation can be interrupted without concerns for systemic embolization.
The successful use of ECMO during pregnancy requires thoughtful preparation, fastidious surgical technique, and a multidisciplinary team approach to critical care. The rewards can be spectacular: How often can we save 2 lives with 1 operation?

\section{Reference}

1. Moore SA, Dietl CA, Coleman DM. Extracorporeal life support during pregnancy. J Thorac Cardiovasc Surg. 2016;151:1154-60. 